Introduction Although colonoscopy is considered the optimal procedure for bowel cancer screening, it remains an imperfect tool for cancer prevention, due to missed adenomas and early cancers. Optimal imaging modalities, innovative scopes and accessories (cap-assisted colonoscopy) have attempted to decrease the adenoma miss rate. Adenoma detection rates (ADR) have been shown to be a key performance indicator

Methods Endocuff-vision is a simple accessory mounted at the end of the scope with a proximal row of $6 \mathrm{~mm}$ length soft plastic, finger-like projections. During scope insertion, these projections invert towards the shaft of the tube and during withdrawal they evert to hold back the colonic folds augmenting the forward endoscopic views. ADRs were recorded and evaluated for screening colonoscopy procedures before and after introduction of Endocuff-vision.

Results To date, four screening endoscopists (BPS, STG, CF, $\mathrm{AH})$ have used the Endocuff-vision as part of a clinical evaluation process form August 2013 until November 2013. From our local Bowel Cancer Screening Program database, the figures of caecal intubation rate (CIR) and the ADRs of the screening endoscopists during April 2013 to July 2013 before Endo-cuff were retrieved:

BPS: CIR-100\%/ADR-62.72\%,

STG: CIR-95.84\%/ADR-40.03\%,

CF: CIR-93\%/ADR-36.76\%,

AH: CIR-96.25\%/ADR- 55.35\%.

Prior to the introduction of the Endocuff-vision, the cumulative CIR was $96.27 \%$ and ADR was calculated to be $48.71 \%$.

The total number of procedures where Endocuff-vision has been mounted was in 30 occasions (BPS-10, STG-11, CF-3, AH6) with similar CIR rates but increased cumulative ADR of $65.5 \%$. On 3 patients the Endocuff-vision was electively removed from the scope due to insertion difficulties through fixed sigmoid colonic segments secondary to severe diverticular disease. There were no adverse events reported during the trial evaluation period.

Conclusion In this small pilot study, use of the Endocuff appeared to improve the ADR by $17 \%$. There were no complications from the use of the cuff although it was electively removed in 3 cases with severe sigmoid colon diverticulosis. Further randomised evaluation of this simple novel device is warranted.

Disclosure of Interest None Declared.

\section{PWE-068 ENDOSCOPIC RESECTION OF COMPLEX COLONIC POLYPS - WHERE DO THE BOUNDARIES LIE?}

T Elliott, ZP Tsiamoulos*, N Suzuki, BP Saunders. Wolfson Unit for Endoscopy, St Mark's Hospital/Academic Institute, London, UK

\subsection{6/gutjnl-2014-307263.328}

Introduction The role of endoscopic resection for colonic polyps previously destined for surgery is expanding. However, surgery remains appropriate in some cases. The aim of this study was to examine tertiary polyp referrals that did not undergo endoscopic polypectomy. The objectives were to determine (i) the proportion of polyps referred for polypectomy that were not endoscopically resected, (ii) the primary reason in this decisionmaking and (iii) factors associated with polyps that were not endoscopically resected.

Methods A prospective observational study of all polyps referred for endoscopic resection (ER) to a tertiary centre between January 2010 and August 2012 was performed. For each case, ER was either completed, abandoned or not attempted. The primary reason for abandoning or not attempting ER was documented. Demographics, polyp characteristics and histology were recorded and a comparative analysis (using chi-square test and independent-samples $\mathrm{T}$ test) was made between patients in whom ER was abandoned or not attempted with those in whom ER was completed.

Results ER was either abandoned (n/29) or not attempted (n/55) in 84 of 423 polyp referrals. This was most commonly because of suspected invasive cancer (45/84). Of these 45 polyps, 12 had characteristic macroscopic features of cancer on inspection. In $24 / 45$, invasive cancer was suspected after advanced endoscopic examination (including surface morphology (Paris/NICE/Kudo) classification and forceps palpation). In 9/45, invasive cancer was only suspected during attempted ER, which was then abandoned. The remaining 41/84 polyps for which ER was abandoned or not attempted appeared benign. The positive and negative predictive values of endoscopic evaluation for the diagnosis of invasive cancer were $86 \%$ and $96 \%$ respectively. The benign-appearing polyps were not endoscopically resected because of (i) a high risk location (ie. overlying the appendix, IC valve or a diverticulum), $\mathrm{n}=12$; (ii) difficult access, $\mathrm{n}=12$; (iii) size $\geq 5 \mathrm{~cm}$ combined with other factors, $\mathrm{n}=8$; (iv) age/ comorbidities, $\mathrm{n}=4$ or (v) poor tolerance of colonoscopy, $\mathrm{n}=$ 2. Forty-six percent of these benign polyps were in the caecum. In comparison with patients who underwent complete ER, those in whom ER was abandoned or not attempted were more likely to be female (56 vs. $37 \%, \mathrm{P}<0.001$ ), had larger mean polyp size $(4.7 \mathrm{~cm}$ vs $3.7 \mathrm{~cm} \mathrm{P}<0.001)$, and had a higher incidence of polyp cancer on histology (47 vs. $2.7 \% \mathrm{P}<0.001$ ).

Conclusion Twenty percent of polyps referred to a tertiary institution for polypectomy may not be suitable for endoscopic resection. This is most commonly due to the presence of invasive cancer which can usually be recognised by endoscopic examination.

Disclosure of Interest None Declared.

\section{PWE-069 COMPARISON OF MICROWAVE WITH MONOPOLAR AND BIPOLAR COAGULATION IN A PORCINE MODEL}

${ }^{1}$ ZP Tsiamoulos*, ${ }^{2} \mathrm{C}$ Hancock, ${ }^{3} \mathrm{PD}$ Sibbons, ${ }^{1} \mathrm{BP}$ Saunders. 'Wolfson Unit for Endoscopy, St Mark's Hospital/Academic Institute, London, UK; ${ }^{2}$ Department of Electronic Engineering, Bangor University, Bangor, UK; ${ }^{3}$ Department of Surgical Sciences, Northwick Park Institute for Medical Research, London, UK

\subsection{6/gutjnl-2014-307263.329}

Introduction Intra-procedural bleeding is considered an immediate serious adverse event and a major concern for the endoscopist and the patient. Current endoscopic devices utilise monopolar or bipolar energy to treat acute bleeding vessels and/ or pre-coagulate visible vessels but there are no ex vivo comparative studies assessing the safety profile with histology.

Methods The optimal time of application for the microwave modality of a new endoscopic device "Speedboat-RS2, Creo Medical Ltd, UK" was initially assessed compared to a standard mono-polar endoscopic device, Coagrasper, Olympus, USA. After histological assessment of the optimal time range, a comparison of the Speedboat RS2 to a standard bipolar endoscopic device, Gold Probe, Boston Scientific, USA, and to standard monopolar device, Coagrasper, was performed to assess the safety profile of coagulation with histology and the endoscopic performance of pre-coagulation in the porcine colon. The Speedboat-RS2 blade delivered microwave coagulation $(5.8 \mathrm{GHz})$ for 
hemostasis. The following parameters were measured in 3 female pigs: histological assessment and pre-coagulation endoscopic performance. All animals were recovered for 2, 5 and 7 days.

Results In animal one, microwave bursts of 5, 10 and $15 \mathrm{sec}$ were applied to the revealed submucosa compared to standard monopolar bursts of a $1 \mathrm{sec}$. Histology showed that 5 and $10 \mathrm{sec}$ of microwave has equivalent histological appearance with standard monolopar preserving the serosal integrity with mild muscle alteration. In animal 2 and 3, microwave was applied for $9 \mathrm{sec}$ in 6 lesions, standard monopolar was applied for 1-2 sec in 6 lesions and standard bipolar was applied for 3-4 sec in 6 more lesions. Histology showed viable serosa with no muscle alterations in microwave group, viable serosa with mild muscle alterations in standard bipolar and viable serosa with mild/moderate muscle alterations in standard monopolar group. In all cases muscle layer cells were contiguous. During the pre-coagulations endoscopic assessment, all modalities were applied to coagulate vessels with median calibre of $2 \mathrm{~mm}$ before and after dissection. Effective pre-coagulation was achieved in 3 out of the 6 visible vessels (microwave group) and in 2 out of the 6 visible vessels (standard monopolar and bipolar groups). Effective coagulation (defined when blood flow stopped) was achieved after the dissection, in all three groups.

Conclusion Compared to Coagrasper (monopolar) and Gold Probe (bipolar), the microwave modality of Speedboat RS2 appears to be equivalent during the pre-coagulation phase. The safety profile of coagulation phase resembles the profile of the other two modalities but with less muscle alterations in the histological specimens.

Disclosure of Interest Z. Tsiamoulos Consultant for: Creo Medical Ltd, C. Hancock Shareholder of: Creo Medical Ltd, P. Sibbons Paid instructor for: Creo Medical Ltd, B. Saunders Consultant for: Creo Medical Ltd, Paid instructor for: Olympus KeyMed.

\section{Inflammatory bowel disease II}

\section{PWE-070 PERSPECTIVES AND ATTITUDES TO COLONOSCOPY IN PATIENTS WITH INFLAMMATORY BOWEL DISEASE}

C Alexakis*, L Medcalf, V Chhaya, J-Y Kang, P Neild, A Poullis, R Pollok. Department of Gastroenterology, St George's Hospital, London, UK

\subsection{6/gutjnl-2014-307263.330}

Introduction Visualisation of mucosa at ileocolonoscopy (IC) remains the gold standard in the assessment of mucosal healing $(\mathrm{MH})$ in patients with inflammatory bowel disease (IBD). $\mathrm{MH}$ is evolving as a key endpoint in assessing response to therapy. This will invariably mean an increased endoscopic burden on these patients. We aimed to investigate IBD patients' perspectives on this, as well as their tolerance of IC.

Methods Consecutive patients attending IBD clinic between September and December 2013 were questioned on their experience of IC. Data on sex, age, disease type (Crohn's (CD) or ulcerative colitis (UC)), duration of illness, and no. of IC was obtained. They were asked to grade various components of the IC experience (concerns about complications, bowel preparation, disruption to life, procedure discomfort, travel to hospital) on a standardised tolerance scale from 1-5. They were also asked to qualitatively rate their overall experience of IC (not unpleasant/ neither unpleasant nor pleasant/bearable/unpleasant/very unpleasant). Finally, they were asked how often they would be prepared to undertake IC in the future.
Results 98 patients responded (46\% male). Mean age was 43.2 years. 33 had UC, 50 had CD, and 11 were unsure of diagnosis. Mean no. IC was 3.7. $62 \%$ had a disease duration $>5$ years, with only $4 \%$ diagnosed in the previous year. Mean tolerance scores for the group were: concerns about complications 2.6, bowel preparation 3.0, disruption to life 2.4 , discomfort during procedure 3.0, travel to/from hospital 1.7. Comparisons between patient subgroups CD versus UC, age $\geq 55$ years versus age $<55$ years, $\geq 4$ IC versus $<4$ IC, and diagnosis $\geq 5$ years versus diagnosis $<5$ years, revealed no significant differences in scores. However, comparison between sexes showed females were more worried about the procedure than males ( 3.0 vs. $2.1, \mathrm{p}=0.02)$, were less tolerant of bowel preparation (3.5 vs. $2.3, \mathrm{p}<0.001)$, experienced more disruption to their lives $(2.9$ vs. $1.9, \mathrm{p}<$ $0.001)$ and were more troubled by travel concerns (2.0 vs. 1.4 , $\mathrm{p}=0.02)$. The majority of the patients felt IC was bearable $(53 \%)$ with only $13 \%$ describing it as very unpleasant. $55 \%$ would have the procedure as frequently as required if their physician felt it appropriate. $7 \%$ would only have IC every 5 years.

Conclusion Bowel preparation and procedural discomfort are the most bothersome aspects of IC amongst IBD patients. There are clear differences in tolerance between female and male patients, and these results should prompt endoscopy units to find ways of improving patient acceptability. This could be provided in the form of a pre-test telephone consultation. A minority of patients were reluctant to increase their frequency of IC, which may be relevant in the monitoring of $\mathrm{MH}$ in their future management.

Disclosure of Interest None Declared.

\section{PWE-071 EFFICACY OF INFLIXIMAB AS SECOND-LINE BIOLOGIC IN CROHN'S DISEASE}

${ }^{1} \mathrm{E}$ Russo*, ${ }^{2} \mathrm{R}$ Hackett, ${ }^{3} \mathrm{~S}$ Campbell, ${ }^{4} \mathrm{JO}$ Lindsay, ${ }^{5} \mathrm{PM}$ Irving, ${ }^{6} \mathrm{TR}$ Orchard. ${ }^{1}$ Medicine, Imperial College London, UK; ${ }^{2}$ Barts and the London School of Medicine and Dentistry, London, UK; ${ }^{3}$ Gastroenterology, MAnchester Royal Infirmary, Manchester, UK; ${ }^{4}$ Gastroenterology, Barts and the London NHS Trust, London, UK; ${ }^{5}$ Gastroenterology, Guy's and St Thomas' Hospitals, London, UK; ${ }^{6}$ Gastroenterology, St Mary's Hospital, London, UK

\subsection{6/gutjnl-2014-307263.331}

Introduction A common therapeutic strategy in events of failure of the first-line anti-TNF $\alpha$ biologic in Crohn's Disease (CD) is to switch to another biologic agent from the same class. There is a significant body of evidence to support using adalimumab (ADA) as second-line therapy following infliximab (IFX) intolerance or loss of response. The aim of this study is to provide evidence around the use of IFX as second-line therapy in CD, an empirical strategy gaining popularity since both agents have recently been licensed as first line agents for moderate-to-severe CD.

Methods Inflammatory Bowel Disease (IBD) specialists from 5 hospitals were invited to contribute all suitable cases through a secure online questionnaire. Data was gathered on patient demographics, disease extent and behaviour, prior or concurrent therapies, duration and outcome of ADA and IFX therapy and reason for switching. Response to the biologic was determined using Physician's Global Assessment (PGA) by each IBD specialist.

Results Data on 28 patients (10 male) were gathered. Median duration of disease before starting biologics was 4.5 years.

Of the 19 patients on ongoing IFX, 5/19 (26\%) are on monotherapy $11 / 19(58 \%)$ are on concurrent immunomodulator 2/19 